\title{
A Study of Determinants of Sensorineural Hearing Loss in Chronic Suppurative Otitis Media with or without Cholesteatoma
}

\author{
Rajwant Kaur ${ }^{1}$, Satinder Pal Singh ${ }^{2 *}$, Jagdeepak Singh ${ }^{1}$ \\ ${ }^{1}$ Department of ENT \& Head Neck Surgery, GMC, Amritsar, India \\ ${ }^{2}$ Department of ENT \& Head Neck Surgery, Swift Hospital, Amritsar, India \\ Email: rajwin000@gmail.com, ${ }^{\star}$ satinderpalsingh44@gmail.com, singhjagdeepak@yahoo.co.in
}

How to cite this paper: Kaur, R., Singh, S.P. and Singh, J. (2018) A Study of Determinants of Sensorineural Hearing Loss in Chronic Suppurative Otitis Media with or without Cholesteatoma. International Journal of Otolaryngology and Head \& Neck Surgery, 7, 148-159.

https://doi.org/10.4236/ijohns.2018.74018

Received: May 19, 2018

Accepted: July 2, 2018

Published: July 5, 2018

Copyright $\odot 2018$ by authors and Scientific Research Publishing Inc. This work is licensed under the Creative Commons Attribution International License (CC BY 4.0).

http://creativecommons.org/licenses/by/4.0/

(c) $\underset{\mathrm{EY}}{\mathrm{C}}$ Open Access

\begin{abstract}
Aim: This study was carried out to assess association between Sensorineural hearing loss (SNHL) and Chronic Suppurative Otitis Media (CSOM), to identify possible risk factors and determine correlation of duration of CSOM and incidence of SNHL. Material and Method: The study consisted of $100 \mathrm{pa}-$ tients of either sex between 15 and 40 years of age, suffering from unilateral CSOM for the past three months or more, the normal ear served as control. Results: This study showed duration of symptoms is $18 \%$ patients for $5-10$ years and below 5 years in $73 \%$ patients. It was found that mean BC thresholds were significantly higher in the diseased ears as compared to the control ears. Though the higher frequencies are more affected than the lower frequencies, in subtotal and attic perforations the speech frequencies seemed to be maximally affected. Conclusion: CSOM should be diagnosed early and should be managed effectively so as to prevent the chances of developing SNHL.
\end{abstract}

\section{Keywords}

CSOM, Cholesteatoma, Sensorineural Hearing Loss (SNHL)

\section{Introduction}

Sensorineural hearing loss (SNHL) in Chronic Suppurative Otitis Media (CSOM) is well documented. In CSOM it can be induced by different mechanisms. SNHL is caused by failure in cochlear transduction of sound from mechanical energy in the middle ear to the neural impulses in the VIII cranial nerve, or by the malfunctioning in the central processing centre of the brain. 
When any one structure of the sensorineural mechanism is impaired by some way, its ability to transduce mechanical energy to electrical energy is reduced resulting in hearing deterioration [1]. Influence of inflammatory factors, mediators of inflammatory reaction, free radicals, or bacterial toxins on cochlear function is also evident [2]. It has been suggested that toxins in CSOM can damage cochlea so in addition to the conductive hearing loss (CHL), SNHL may occur as a result of CSOM [3].

In CSOM toxins enter through the semipermeable round window membrane causing damage to organ of Corti. It has been suggested that temporary threshold shifts occurred because of serous labyrinthitis, while permanent threshold shifts occurred from permanent dysfunction of the organ of Corti. The anatomical characteristics of the round window are such that it encourages the accumulation, stagnation and absorption of purulent secretions into the perilymph [4].

The round window membrane has 3 layers which together measure approximately $0.065 \mathrm{~mm}$. The round window niche is approximately $1 \mathrm{~mm}$ in depth and $2 \mathrm{~mm}$ in diameter. There are essentially no ciliated cells in the region of the round window under normal circumstances. Pus can be pooled in the adjacent sinus tympani space, especially when the patient is in an upright position. Remnants of mesenchyme in the round window niche and sinus tympani are also slow to be reabsorbed. These factors encourage pus or infected tissue to be concentrated at the round window thereby encouraging absorption through the round window leading to chemical contamination of the perilymph [5].

Many studies were conducted to find out the relationship between SNHL and CSOM. A cross sectional study conducted by Borlingegowda Viswantha et al. on 150 patients of age group 10 to 50 years, having CSOM squamous type, which could be retraction pocket or an attic or marginal perforation. Only the duration of disease was found to have a significant correlation with increasing incidence of SNHL as incidence increase especially after 5 years of the disease. The value was noted to be 0.018 [6].

In another retrospective study done by Azevedo et al. with 115 patients with CSOM with and without cholesteatoma, the average duration of ear discharge was 12.4 years. SNHL occurred only in 13\% of patients of CSOM, and correlated with older age, but not with the presence of cholesteatoma or duration of ear disease [7].

The present study was carried out to study the determinants of SNHL in patients with CSOM with or without cholesteatoma with the objectives, to assess the association between SNHL and CSOM, to investigate clinical factors that affect the sensorineural component in patients with this disease, to report the prevalence, type, and severity of hearing loss in CSOM and identify risk factors, and to study the correlation of duration of disease with the incidence of SNHL.

\section{Material and Methods}

\section{Study design}

This is a retrospective study conducted between March 2014-16 with a target 
population of one hundred successive adults of either sex, between 15 to 40 years of age seen in the Ear Nose and Throat (ENT) clinics of the Government Medical College (GMC), Amritsar, suffering from unilateral CSOM for the past three months or more were included in the study with tympanic membrane perforations.

\section{Study location}

Department of ENT, Government Medical College (GMC), Amritsar, is a tertiary health institution and a major referral center in Punjab, located within North India.

\section{Sample size}

One hundred successive adults of either sex, between 15 and 40 years of age seen in the Ear Nose and Throat (ENT) clinics.

\section{Sampling techniques}

Approval of the Ethical Committee of Government Medical College, Amritsar was also obtained. All consecutive adults seen in the ENT clinic within the three year period were screened for tympanic membrane perforations. Predetermined inclusion and exclusion criteria were applied while recruiting patients in the study. Subjects above the age of 40 years were not included so as to exclude persons with possible effects of ageing process on hearing. A prior informed consent from all patients was obtained from each patient with tympanic membrane perforations.

This study was carried out to assess association between SNHL and CSOM, to identify possible risk factors and determine correlation of duration of CSOM and incidence of SNHL.

\section{INCLUSION CRITERIA}

1) Unilateral CSOM (normal tympanic membrane in contralateral ear). This normal contralateral ear will serve as a control because it eliminates variables such as noise, hereditary or congenital causes \& presbyacusis.

\section{EXCLUSION CRITERIA}

1) History of previous otologic surgery

2) History of familial hearing loss

3) Previous exposure to ototoxic drugs, positive fistula test, Frank labyrinthitis

4) History of habitual exposure to noise, head trauma, meningitis

\section{Data collection}

Each of the participants was interviewed with a pre tested structured questionnaire and complete ENT examination was carried out in each patient with particular reference to the otoscopic findings. Nose and throat were examined to exclude any associated pathology. Ear drum was inspected on both sides suction under microscope was carried out wherever necessary to establish the type of tympanic membrane pathology. Based on the findings of tympanic membrane, patients were divided into three groups:

- Group I-Patients with Central perforation (safe pathology)

- Group II-Patients with marginal or posterosuperior perforation 
- Group III-Patients with attic pathology

\section{Diagnostic criteria of SNHL}

Tuning fork tests (Rinne's, Weber's and Absolute Bone Conduction tests) were done using 256, 512 and, $1024 \mathrm{~Hz}$ frequencies in each patient. A note was also made of any findings of spontaneous or positional nystagmus wherever present. Audiological evaluation included Pure Tone Audiometry (PTA), Speech Audiometry, and Impedance Audiometry.

The patients' hearing levels in decibel were assessed with a biologically calibrated AA 222 diagnostic audiometer. The BC threshold at the affected side were measured at a frequency of 500,1000, 2000, and $4000 \mathrm{~Hz}$ respectively in an acoustically treated sound proof boot and compared with those in the unaffected ear. Air and bone conduction thresholds were determined. The Mean hearing loss was calculated through the pure tone average taken at $500 \mathrm{~Hz}, 1000 \mathrm{~Hz}$ and $2000 \mathrm{~Hz}$ for each site of perforations (1 to 5).

The data so obtained was collected, collated and analysed using Chai square and Pearson's tests. The t-test was applied were appropriate. The level of significance was determined and the $\mathrm{p}$ value of $<0.05$ was taken as statistically significant.

- $\mathrm{p}$ value $>0.05$ difference is not significant

- $\mathrm{p}$ value $<0.05$ difference is significant

- $\mathrm{p}$ value $<0.001$ difference is highly significant

\section{Results}

The present study was conducted in 100 patients suffering from CSOM and out of which, $65 \%$ were females and $35 \%$ males giving male to female ratio of $1: 2$ (Table 1).

It was observed that largest group (42\%) comprised of patients in 31 - 40 years age group followed by the group of patients aged between $21-30$ years. The smallest group consisted of patients in $10-20$ years age group (23\%). The mean age is $28.72 \pm 8.68$ years (Graph 1 ).

More than half of the patients (57\%) showed involvement of left ear. All the patients included in the study had history of ear discharge and hearing loss. This hearing loss was of a variable degree. Another key symptom was earache (7\%) followed by tinnitus (4\%). In nearly three-fourth patients the symptoms of ear disease were present for the first five years. Duration of symptoms in $18 \%$ patients these were present for more than five years but less than ten years and 73\%

Table 1. Gender wise distribution of patients.

\begin{tabular}{ccc}
\hline Gender & No. of patients & \% age \\
\hline Male & 35 & 35.0 \\
Female & 65 & 65.0 \\
Total & 100 & 100.0 \\
\hline
\end{tabular}


of patients presented within first five years of onset of disease. Most of the patients (81\%) had history of moderate ear discharge while another $18 \%$ had scanty ear discharge. Amongst most of the patients (96\%) the ear discharge did non-foul smell.

Age, Gender, and duration of ear discharge were three risk factors to develop mixed type of hearing loss in patients included in this study. Almost two-third patients who had MHL were female. Similarly, $80 \%$ of patients with MHL were in the 30 to 40 years age group. Yet another risk factor identified was duration of ear discharge. In $42 \%$ patients with MHL, the ear discharge was present for the last five years. In half of these patients, the ear discharge was seen for more than 10 years (Graph 2).

Majority of patients (91\%) presented with central perforation of the tympanic membrane while $9 \%$ patients showed unsafe type of pathology on otoscopic examination. In four-fifth of patients with safe type of middle ear pathology, the

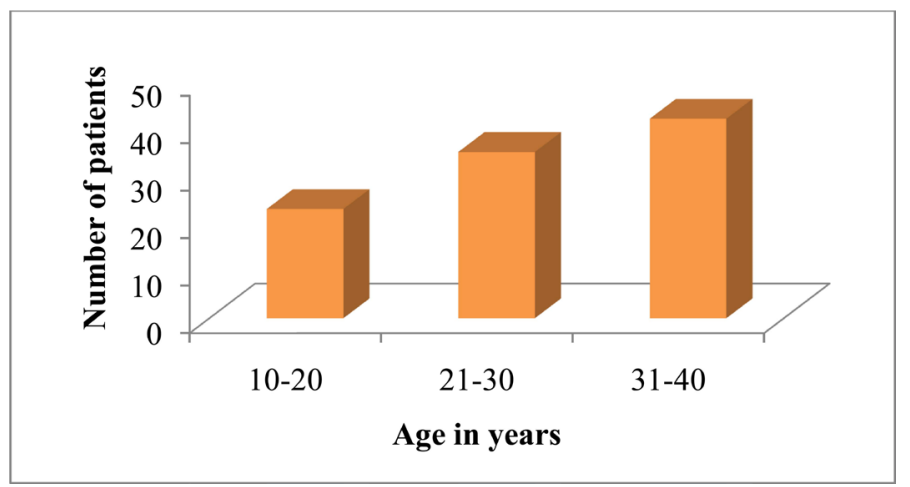

Graph 1. Distribution of patients according to their age.

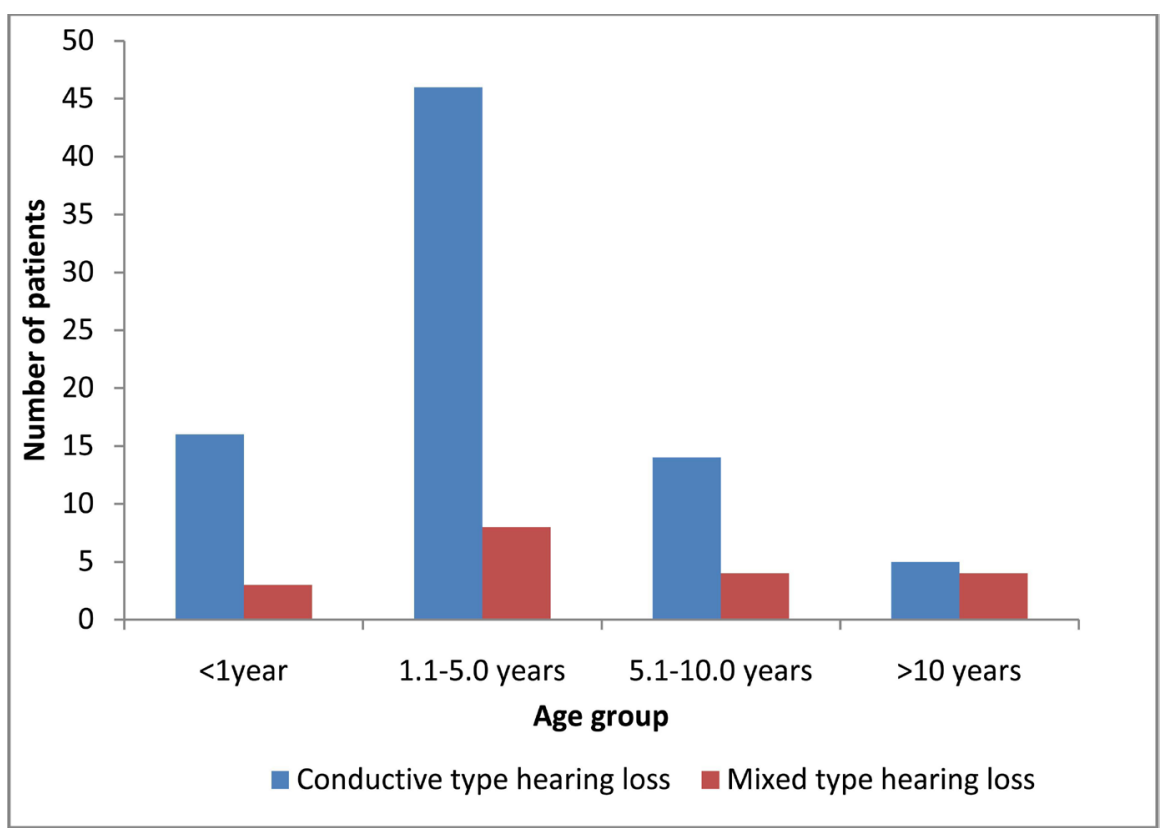

Graph 2. Showing distribution of patients according to duration of ear discharge in conductive and mixed type hearing loss. 
hearing loss was conductive in nature.

A total of 91 patients were diagnosed with safe type of middle ear pathology. Out of these, 73 patients $(80 \%)$ had conductive hearing loss. Similarly, in patients with unsafe type of ear disease, almost $90 \%$ had conductive hearing loss. Nine patients included in the present study had unsafe type of middle ear pathology. Out of these, 8 (88\%) had conductive hearing loss (Graph 3).

An attempt was made to find out any relationship between type of middle ear pathology (as evidenced by otoscopic findings) and nature of hearing loss (conductive or mixed). In the present study a total of 81 patients had a conductive hearing loss. On the basis of otoscopic findings seventy-three (90.12\%) patients were diagnosed to be suffering from a safe type of CSOM, while eight (9.88\%) patients were diagnosed to be having unsafe type of CSOM. Another 19 patients had a mixed type of hearing loss. Clinically, $94.73 \%$ patients had safe type of CSOM, whereas only $5.27 \%$ had unsafe type of pathology.

A total of 91 patients presented with safe type of middle ear pathology. Out of them, 18 patients $(20 \%)$ had mixed type of hearing loss. Out of nine patients with unsafe type of middle ear pathology, one patient had mixed type of hearing loss. Out of 19 patients who had mixed type of hearing loss, most of them had safe type of disease. The results of present study suggest that majority of patients of CSOM with mixed hearing loss develop moderate to profound grade of hearing loss. In this study, amongst 19 patients who had mixed type of hearing loss, the grade was mild in only $21 \%$ subjects. While similar $26.35 \%$ losses were seen in moderate to moderately severe and profound hearing loss respectively.

Most of the patients had a B Type of graph on impedance audiometry on diseased side. While in control ears it was A Type.

Amongst the 100 control ear with normal otoscopic findings, 8 patients had SNHL. 3 patients had moderate degree, 3 had mild degree, and the rest 2 patients

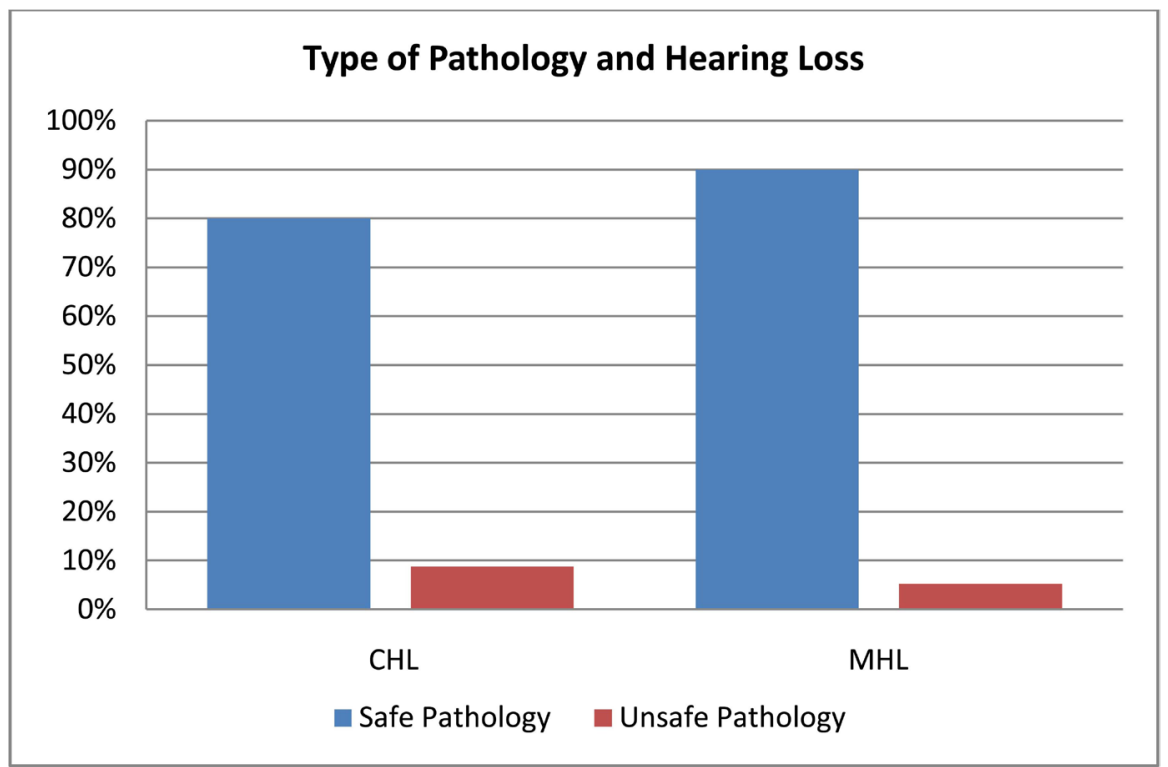

Graph 3. Type of pathology and hearing loss. 
had minimal degree of SNHL.

When the pure tone average (PTA Av) were compared between diseased and control ear, in patients with pure conductive hearing loss mean PTA Av was $29.40 \mathrm{~dB}$ in diseased and $15.95 \mathrm{~dB}$ in control ears. In patients with mixed type of hearing PTA Av was $61.96 \mathrm{~dB}$ in diseased as compared to $31.32 \mathrm{~dB}$ in control ears.

Mean BC threshold of both the diseased and control ear for 0.5, 1, 2 and 4 $\mathrm{KHz}$ frequencies were analysed and it was found that mean $\mathrm{BC}$ threshold were significantly higher in the diseased ears as compared to the control ears. The mean BC threshold difference of the diseased and control ear at $0.5,1,2$, and 4 $\mathrm{KHz}$ frequencies was observed to be 1.05, 1.9, 3.95, $4.45 \mathrm{~dB}$ respectively. So it was significantly higher at 2 and $4 \mathrm{KHz}$ frequencies (Table 2).

The bone conduction thresholds of patients with mixed type of hearing loss were compared with those in control ears at $0.5,1,2$, and $4 \mathrm{KHz}$. The mean of bone conduction thresholds in ears with mixed type of hearing loss at $2 \mathrm{KHz}$ was $30 \mathrm{~dB}$, while in control ears it was $20 \mathrm{db}$ (Table 3 ).

\section{Discussion}

CSOM tubotympanic variety is often considered as safe variety without the involvement of inner ear. It can lead to middle ear changes which include irreversible mucosal changes, presence of granulation tissue, cholesteatoma formation, tympanosclerosis and ossicular destruction [8]. It has been postulated that SNHL commonly occurs in patients with CSOM [9]. We have observed definite and significant correlation between duration of disease (chronic suppurative otitis media) and sensorineural hearing loss.

The incidence of mixed type of hearing loss in the present study was $19 \%$. Twenty percent of patients with safe type of middle ear pathology had a mixed

Table 2. Showing average BC threshold at different frequencies

\begin{tabular}{ccccc}
\hline Frequency & Control ear & Diseased ear & Difference & P value \\
\hline $0.5 \mathrm{KHz}$ & $13.15 \pm 9.47$ & $14.20 \pm 8.98$ & 1.05 & 0.30 \\
$1 \mathrm{KHz}$ & $11.95 \pm 8.34$ & $13.85 \pm 7.38$ & 1.9 & $0.03^{*}$ \\
$2 \mathrm{KHz}$ & $14.90 \pm 12.03$ & $18.85 \pm 10.63$ & 3.95 & $0.001^{\star *}$ \\
$4 \mathrm{KHz}$ & $16.60 \pm 11.36$ & $21.05 \pm 12.83$ & 4.45 & $0.001^{\star *}$ \\
\hline
\end{tabular}

$\mathrm{N}=100 ;{ }^{*}$ (Significant), ${ }^{* *}($ Highly Significant $)$.

Table 3. Mean audiometric thresholds.

\begin{tabular}{cccc}
\hline Variable & Control ear & Diseased ear & P value \\
\hline Mean AC threshold & $21.75 \pm 13.90$ & $41.16 \pm 14.92$ & 0.001 \\
Mean BC threshold & $14.15 \pm 9.44$ & $16.98 \pm 8.74$ & 0.001 \\
AB gap mean & $7.60 \pm 7.33$ & $24.17 \pm 10.34$ & 0.001 \\
\hline
\end{tabular}

$\mathrm{N}=100, \mathrm{p}<0.001$. 
type of hearing loss, while only one out of nine patients (5.27\%) with unsafe ear disease was diagnosed with MHL. Thus based on the results of present study it appears that unsafe type of middle ear pathology is not a risk factor for developing mixed type of hearing loss in CSOM. However age, gender, and duration of ear discharge are probable risk factors. Similar study enrolled by Nanda et al, to assess the association between SNHL and safe mucosal CSOM and its relation to patients age, sex, duration of disease, active or inactive disease, and speech frequencies on 100 patients with unilateral mucosal type of CSOM with normal contralateral ear, it was observed that there was much higher incidence of SNHL in study ears with CSOM (21\%) compared to control contralateral ears (5\%) without infection. The incidence also increased with age of patient and duration of disease [10]. According to Kholmatov et al., duration of disease when compared with incidence of SNHL, a progressively high incidence of SNHL was found as the duration of the disease increased [11].

Yet another key finding of present study was that majority of patients with safe type of middle ear pathology tend to develop a CHL which is mild in grade. However, when patients developed MHL, it tends to be moderate to moderately severe in nearly half of the patients. A small percentage of patients develop profound hearing loss as well.

In the present study, 19\% patients had mixed type of hearing loss i.e. both conductive and SNHL. Patients, in whom, the BC threshold in 250,500, 1000, and $2000 \mathrm{~Hz}$ frequencies were $25 \mathrm{~dB}$ or higher were considered to be having SNHL. A number of studies have used different benchmarks to identify SNHL based on $\mathrm{BC}$ thresholds and have reported divergent figures of incidence of SNHL in patients with CSOM (safe or unsafe type) [12] [13] [14] (Table 4).

It is evident from above table that there is variance in the reported incidence of SNHL in patients of CSOM. With $15 \mathrm{~dB}$ benchmark a study reported this incidence as high as $43 \%$, however when the same study changed the benchmark to $30 \mathrm{~dB}$, this incidence fell to $16 \%$ [9]. Yet, another study which also used $30 \mathrm{~dB}$ as a benchmark reported an incidence of $13 \%$ [7]. However most of the studies

Table 4. The incidence of SNHL in various studies.

\begin{tabular}{ccc}
\hline Study & BC Threshold used to label SNHL & Incidence of SNHL in CSOM \\
\hline Paparella et al. & $15 \mathrm{Db}$ & $43 \%$ \\
Sharma et al. & $25 \mathrm{~dB}$ & $9.4 \%$ \\
Levine et al. & $25 \mathrm{~dB}$ & $12 \%$ \\
Mohsin et al. & $25 \mathrm{~dB}$ & $15.09 \%$ \\
Viswanatha et al. & $25 \mathrm{~dB}$ & $16.05 \%$ \\
Kaur et al. & $25 \mathrm{~dB}$ & $24 \%$ \\
Paparella et al. & $30 \mathrm{~dB}$ & $16 \%$ \\
Azevedo et al. & $30 \mathrm{~dB}$ & $13 \%$ \\
Present Study & $25 \mathrm{~dB}$ & $19 \%$ \\
\hline
\end{tabular}


(including present study) have used a BC threshold of $25 \mathrm{~dB}$ to label it as SNHL. In these studies, the reported incidence of SNHL in CSOM (whether safe, unsafe or both), ranges between $9.4 \%$ and $24 \%$. Thus the incidence of $19 \%$ reported by present study is consistent with similar studies on the subject.

An attempt was made to find out any relationship between type of middle ear pathology (as evidenced by otoscopic findings) and nature of hearing loss (conductive or mixed). The results indicate that unsafe type of middle ear pathology is not a risk factor for development of SNHL. A study which examined the relationship between SNHL and presence or absence of cholesteatoma concluded that CSOM with or without cholesteatoma may evolve towards SNHL (which was observed in $13 \%$ of the cases in this study) [7]. This correlates well with the findings of the present study (Graph 4).

Our study coincides with Levine [14] where there was consistent correlation between severity of sensorineural hearing loss, presence of cholesteatoma and erosion of ossicular chain. The studies of J P Noordzij [3], Macandie \& O’ Reilly [1] we have observed definite and significant correlation between duration of disease and sensorineural heating loss, coinciding with observations of Cusimano [15] and Dumich [16].

The results of present study are in conformity with the earlier studies, that unsafe type of middle ear pathology is not a risk factor for developing SNHL in CSOM. However, age and duration of ear discharge are the probable risk factors and in terms of $\mathrm{BC}$ threshold; $2 \mathrm{KHz}$ and $4 \mathrm{KHz}$ are the most commonly affected on higher frequencies (Table 5).

These differences tended to increase with increasing frequency and were all significant $(\mathrm{P}<0.05)$. So after analysing all these studies it was found that the differences in mean BC threshold of this present study were very much significant.

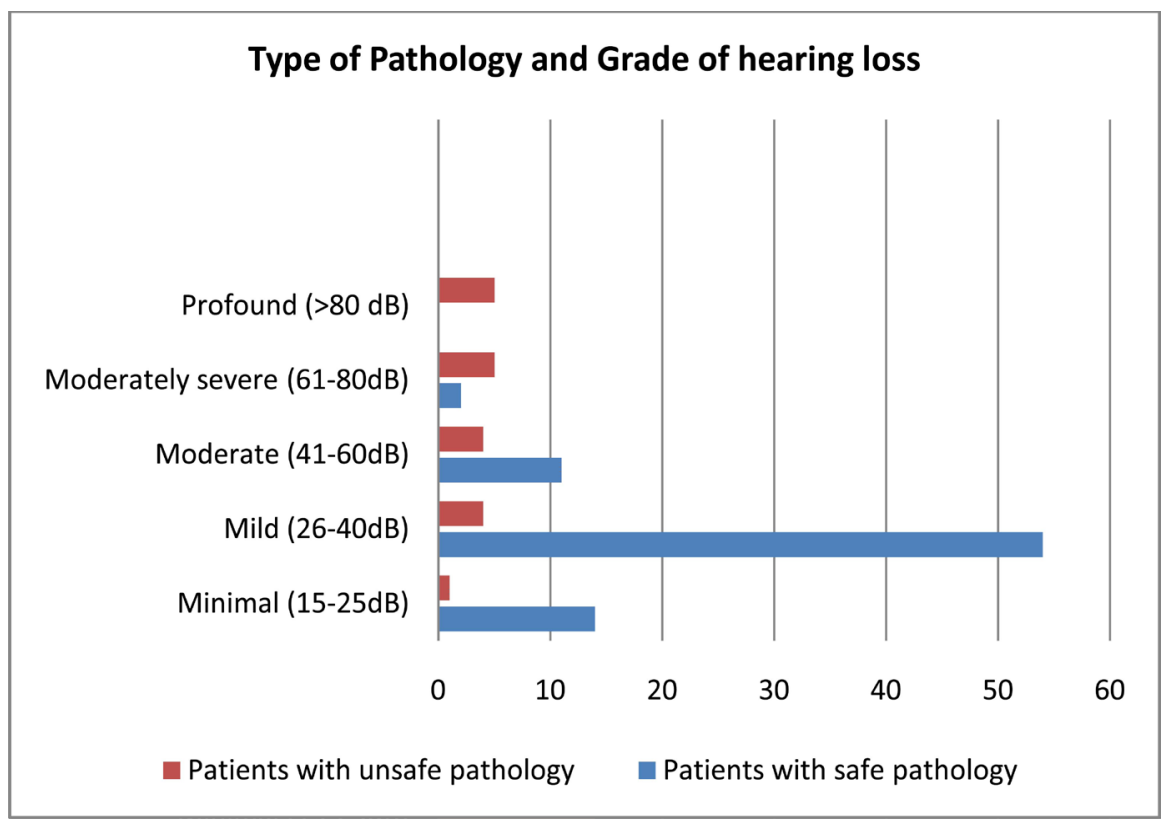

Graph 4. Type of pathology and grade of hearing loss. 
Table 5. Mean BC threshold difference between diseased and control ear/study on different frequencies

\begin{tabular}{ccccc}
\hline $\begin{array}{c}\text { Mean BC threshold difference between } \\
\text { diseased and control ear/study }\end{array}$ & $0.5 \mathrm{KHz}$ & $\mathbf{1 ~ K H z}$ & $2 \mathrm{KHz}$ & $\mathbf{4 ~ K H z}$ \\
\hline Ahmed et al. & $4.1 \mathrm{~dB}$ & $6.0 \mathrm{~dB}$ & $10.7 \mathrm{~dB}$ & $9.7 \mathrm{~dB}$ \\
Chatterji et al. & $0.61 \mathrm{~dB}$ & $4.33 \mathrm{~dB}$ & $3.64 \mathrm{~dB}$ & $1.71 \mathrm{~dB}$ \\
Sharma et al. & $2.6 \mathrm{~dB}$ & $3.4 \mathrm{~dB}$ & $4.4 \mathrm{~dB}$ & $1.8 \mathrm{~dB}$ \\
Noordzig et al. & $-0.5 \mathrm{~dB}$ & $0.9 \mathrm{~dB}$ & $4.4 \mathrm{~dB}$ & $3.6 \mathrm{~dB}$ \\
Kolo et al. & $21.69 \mathrm{~dB}$ & $25.66 \mathrm{~dB}$ & $32.28 \mathrm{~dB}$ & $34.63 \mathrm{~dB}$ \\
Present study & $1.05 \mathrm{~dB}$ & $1.9 \mathrm{~dB}$ & $3.95 \mathrm{~dB}$ & $4.45 \mathrm{~dB}$ \\
\hline
\end{tabular}

\section{Conclusion}

Sensorineural hearing loss (SNHL) in CSOM may be induced by different risk factors reported by present study. The duration and severity of disease, presence of cholesteatoma and bony erosion ossicular chain have been implicated as possible predictive factors associated SNHL. Thus early diagnosis and effectively management of CSOM help to prevent the chances of developing SNHL.

\section{Summary}

The study was conducted in a tertiary centre patients presented in OPD had CSOM with SNHL may be induced by duration and severity of disease, presence of cholesteatoma and bony erosion ossicular chain and it was observed that $19 \%$ of the patients were reported with SNHL in CSOM based on BC threshold mainly affected on higher frequencies.

\section{Limitation of the Study}

This includes the sample size of hundred patients to explain all the factors in SNHL and study was conducted in short duration period.

\section{Acknowledgements}

This research was supported by ENT department, Government Medical College, Amritsar, Punjab. I thank this institute who provided insight and expertise that greatly assisted the research. I would also like to show my gratitude to Dr. Jagdeepak Singh, Professor and Head of ENT department, GMC, Amritsar, Punjab, India.

\section{References}

[1] Ozel, H.E., Kahraman, E., Genc, S. and Cingi, C. (2012) Profound Sensorineural Hearing Loss: Analysis of 310 Adults Cases. B ENT, 8, 173-178.

[2] Jesic, S.D., Jotic, A.D. and Babic, B.B. (2012) Predictors for Sensorineural Hearing Loss in Patients with Tubotympanic Otitis, Cholesteatoma and Tympanic Membrane Retractions. Otology \& Neurotology, 33, 934-940. 
[3] Noordzij, J.P., Dodson, E.E., Ruth, R.A., Arts, H.A. and Lamberts, P.R. (1995) CSOM and Sensorineural Hearing Loss: Is There a Clinically Significant Relation? American Journal of Otolaryngology, 16, 420-423.

[4] Paparella, M.M. and Brady, D.R. (1970) SNH Loss in CSOM and Mastoiditis. Transactions-American Academy of Ophthalmology and Otolaryngology, 74, 108-115.

[5] Kaur, K., Sonkhya, N. and Bapna, A.S. (2003) Chronic Suppurative Otitis Media And Sensorineural Hearing Loss: Is There A Correlation? Indian Journal of Otolaryngology and Head \& Neck Surgery, 55, 1.

[6] Viswanatha, B., Sadasivan, S.S., Vijayashree, M.S. and Ravikumar, R. (2014) Sensorineural Hearing Loss in Squamous Type of Chronic Otitis Media. Indian Journal of Otolaryngology, 3, 41-45.

[7] Azevedo, A.F., Pinto, D.C.G., Souza, N.J.A., Greco, D.B. and Goncolves, D.U. (2007) Sensorineural Hearing Loss in Chronic Suppurative Otitis Media with and without Cholesteatoma. Brazilian Journal of Otorhinolaryngology, 73, 671-674. https://doi.org/10.1016/S1808-8694(15)30128-2

[8] Moshin, M.A., Kumar, M.R., Byrareddy, G.N. and Ravikumar, D. (2013) Sensorineural Hearing Loss in Chronic Suppurative Otitis Media of Tubotympanic Variety. National Journal of Otorhinolaryngology and Head and Neck Surgery, 1, 1-4.

[9] Paparella, M.M., Morizono, T., Le, C.T., et al. (1984) SNHL in Otitis Media. Annals of Otology, Rhinology \& Laryngology, 93, 623-629. https://doi.org/10.1177/000348948409300616

[10] Nanda, M.S. and Luthra, D. (2015) Sensorineural Hearing Loss in Patients with Unilateral Safe Chronic Suppurative Otitis Media. International Journal of Research in Medical Sciences, 3, 551-555. https://doi.org/10.5455/2320-6012.ijrms20150304

[11] Kholmatov, D.I. (2001) Early Detection of a Sensorineural Aspect of Hypoacusis in Patients with Chronic Purulent Otitis Media. Vestnik otorinolaringologii, 3, 26-28.

[12] Sharma, K., Gulati, S.K. and Kaur, K. (2006) Sensorineural Hearing Loss in Chronic Suppurative Otitis Media-Safe Type. Indian Journal of Otology, 11, 22-26.

[13] Viswantha, B., Naseerudin, K., Ravikumar, R., Vijayashree, M.S. and Krishna, N. (2014) Sensorineural Hearing Loss in Complicated Cholesteatoma Ear Disease. Research in Otolaryngology, 3, 29-35.

[14] Levine, B.A., Shelton, C., et al. (1989) Sensorineural Hearing Loss in Chronic Otitis Media, Is It Clinically Significant? Archives of Otolaryngology_Head \& Neck Surgery, 93, 623-629.

[15] Cusimano, F., Cocita, V.C. and D’Amico, A. (1989) Sensorineural Hearing Loss in Chronic Otitis Media. The Journal of Laryngology \& Otology, 103, 158-163. https://doi.org/10.1017/S0022215100108333

[16] Dumich, P.S. and Hamer, S.G. (1983) Cochlear Function in Chronic Otitis Media. Laryngoscope, 93, 583-586. https://doi.org/10.1002/lary.1983.93.5.583 


\section{Abbreviations}

Sensorineural hearing loss (SNHL)

Conductive hearing loss (CHL)

Mixed hearing loss (MHL)

Chronic Suppurative Otitis Media (CSOM)

Air Conduction (AC)

Bone Conduction (BC)

Pure Tone Average (PTA Av) 